\title{
Towards Metaphors for Cascading AI
}

\author{
Jonas Oppenlaender ${ }^{1}$ and Jesse Josua Benjamin ${ }^{2}$ \\ 1 University of Oulu, Oulu, Finland \\ jonas.oppenlaender@oulu.fi \\ 2 University of Twente, Enschede, Netherlands \\ $j \cdot j \cdot$ benjamin@utwente.nl
}

Artificial Intelligence (AI) is becoming more and more ubiquitous. Much of this computing power is provided in the form of an opaque "black box." Calls for AI systems to explain their decisions to users have been growing, and Explainable AI (XAI) is now a flourishing area of research.

AI systems can, as in the case of autonomous vehicles and robots, be situated and embedded in a context that requires interaction with both humans, inanimate objects, and other AI systems. The user of such an AI system, however, primarily interacts with one given AI system at a time through a purposely designed interface. This interface may provide the user with explanations of AI decisions to instil trust (or other sought-after values, e.g. fairness and transparency) in the user of the system. However, much of the research on XAI focuses on the "last mile" - that is, how a single AI system can provide explanations on its decisions to a single user. Today, research on AI transparency and explainability in communities such as CHI, FAccT or ICML is predominantly focused on only one level of explanations at a time. This may be 1) counterproductive for the design and development of effective Human-AI Interaction (HAI) and 2) methodologically inadequate with regards to how socio-technical systems both now and in the future incorporate multiple AI pipelines.

In the future, more and more systems will be powered by AI. This may exacerbate existing blind spots in explainability research, such as focusing on outputs of an individual AI pipeline as opposed to a holistic and integrative view on the system dynamics of data, algorithms, stakeholders, context and their respective interactions. Taking the long view, not only will this intensify the complex interrelationships between an AI system and its "algorithmic others" [1] of datasets or user models that require explanation, but AI systems will increasingly rely on patterns and models of other AI systems. This will likely introduce a major shift in the desiderata of interpretability, explainability and transparency.

In this world of Cascading $\boldsymbol{A I}$ (CAI), AI systems will use the output of other AI systems as their inputs. The typical formulations of desiderata for explaining AI decision-making, such as post-hoc interpretability $[5,6]$ or modelagnostic explanations (e.g., [7]), may simply not hold in a world of cascading AI: any explanation for an object recognition, for instance, will by necessity need to expand over prior and posterior algorithmic transformations such as a) classifications that have led to the selection of this recognition model and/or b) predictions that will be made based on it. Typical interpretability techniques such as feature relevance or uncertainty visualisations would have to become much more entangled with the wider, processual relationships of Cascading AI rather 
than explaining the outputs of a singular model. In this scenario, predominantly focusing on "the last mile" may be shortsighted, for example resulting in a reliance on anthropocentric assumptions that limit the design and engineering of robotics (cf. [2]).

The scenario of AI cascades raises the question of how a user-facing AI system that draws on several other systems should provide explanations to its users? How can transparency on AI decisions be provided in such a scenario? Should one system summarise the explanations of other systems? Is it necessary for all participating AI systems to explain their decisions to the user? Does a trusting, social relationship with AI necessarily need to include all layers, or only the one that the user is directly interacting with? And how far does a social relationship between the user and AI reach down the stack of the cascade?

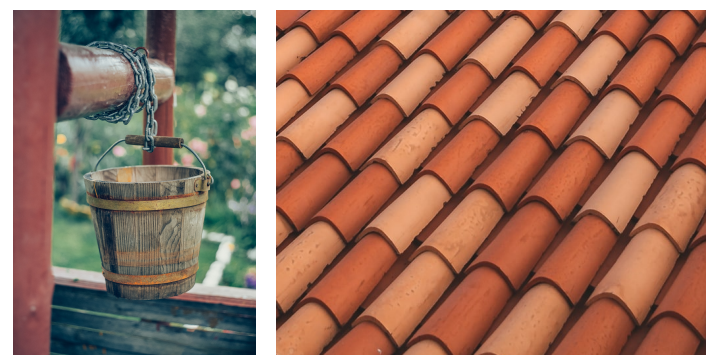

Fig. 1. Metaphors for Cascading AI: water well (left) and imbricated roof tiles (right).

\section{Metaphors for Cascading AI}

Metaphors provide meaning and influence how we experience AI [4]. In our workshop contribution, we propose two metaphors which may help designers to frame their efforts when designing cascading AI systems.

One may view the explanations provided by a user-facing Cascading AI system through the metaphor of a water well. The AI system could provide aggregate explanations (water) to its users by drawing on explanations from other AI systems (ground water). The way explanations are provided is akin to hoisting a bucket full of water (i.e., selected explanations drawn from other AI system) up the well to the end user.

Considering flows of interaction in a situated human-CAI encounter, media theorist Hansen's notion of imbrication [3] can offer a fruitful lens for designers: inasmuch as roof tiles are imbricated, cascading AI systems both overlap and co-extend with how users engage the world. Imbrication is particularly accurate regarding the challenges of CAI, as it takes shape both irregularly (e.g., full conscious overlap is not a given due to opacity) and cumulatively (e.g., overlap in combined human-CAI capacities and agency may still occur). Dissecting the stack of a cascade, then, for the various implicit and explicit human-CAI overlaps could offer pointers to 'fault lines' in interpretability. For instance, a self-driving car system using image recognition, reinforcement learning as well as natural language processing could be analysed for which specific AI technology most significantly 'cascades into' situated human interaction. 


\section{References}

1. Dourish, P.: Algorithms and their others: Algorithmic culture in context. Big Data \& Society 3(2), 2053951716665128 (2016). https://doi.org/10.1177/2053951716665128

2. Drrenbcher, J., Lffler, D., Hassenzahl, M.: Becoming a robot - Overcoming anthropomorphism with techno-mimesis. In: Proceedings of the 2020 CHI Conference on Human Factors in Computing Systems. pp. 1-12. CHI '20, Association for Computing Machinery, Honolulu, HI, USA (Apr 2020). https://doi.org/10.1145/3313831.3376507

3. Hansen, M.B.: Feed-Forward: On the Future of Twenty-First Century Media. No. 2, University of Chicago Press, Chicago, IL (2015)

4. Khadpe, P., Krishna, R., Fei-Fei, L., Hancock, J.T., Bernstein, M.S.: Conceptual metaphors impact perceptions of human-ai collaboration. Proc. ACM Hum.Comput. Interact. (CSCW) (2020). https://doi.org/10.1145/3415234

5. Lipton, Z.C.: The mythos of model interpretability: In machine learning, the concept of interpretability is both important and slippery. Queue 16(3), 3157 (Jun 2018). https://doi.org/10.1145/3236386.3241340

6. Miller, T.: Explanation in artificial intelligence: Insights from the social sciences. Artificial Intelligence 267, 1-38 (2019). https://doi.org/10.1016/j.artint.2018.07.007

7. Ribeiro, M.T., Singh, S., Guestrin, C.: Model-Agnostic Interpretability of Machine Learning. arXiv:1606.05386 [cs, stat] (Jun 2016), arXiv: 1606.05386 